\title{
Correction: Bacterial recognition by PGRP-SA and downstream signalling by Toll/DIF sustain commensal gut bacteria in Drosophila
}

Shivohum Bahuguna, Magda Atilano, Marcus Glittenberg, Dohun Lee, Srishti Arora, Lihui Wang, Jun Zhou, Siamak Redhai, Michael Boutros, Petros Ligoxygakis

Fig 6 is incorrect. The authors have provided a corrected version here.

\section{G open Access}

Citation: Bahuguna S, Atilano M, Glittenberg M, Lee D, Arora S, Wang L, et al. (2022) Correction: Bacterial recognition by PGRP-SA and downstream signalling by Toll/DIF sustain commensal gut bacteria in Drosophila. PLoS Genet 18(2): e1010082. https://doi.org/10.1371/journal. pgen.1010082

Published: February 23, 2022

Copyright: ๑ 2022 Bahuguna et al. This is an open access article distributed under the terms of the Creative Commons Attribution License, which permits unrestricted use, distribution, and reproduction in any medium, provided the original author and source are credited. 


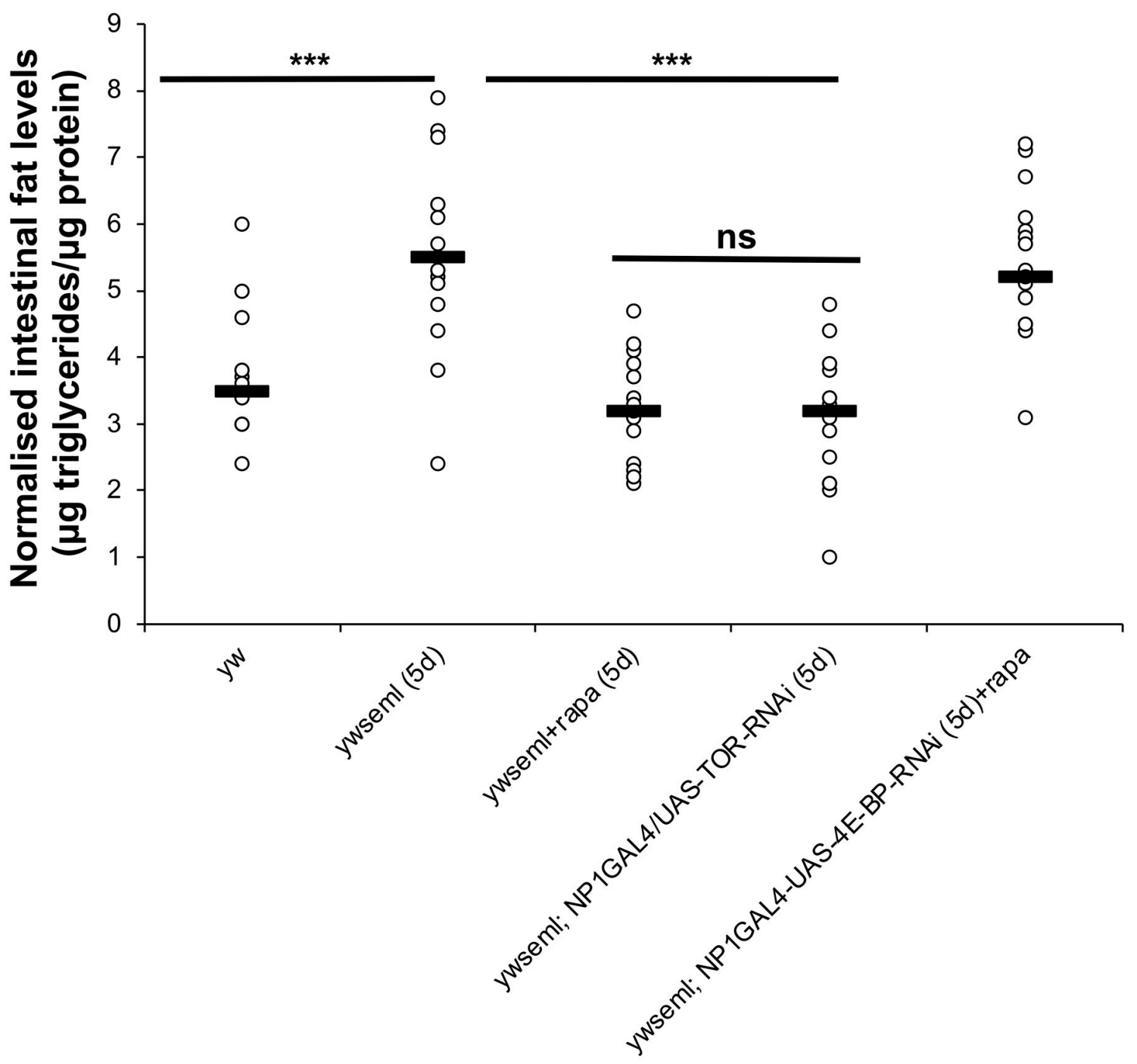

Fig 6. Loss of PGRP-SA increases intestinal fat levels. Loss of PGRP-SA increased intestinal triglyceride levels in 5-day old flies. This phenomenon was suppressed with pharmacological inhibition (rapamycin) or RNAi against TOR in ECs. This was dependent on $4 \mathrm{EBP}$ as $y w \mathrm{seml}$; NP1>4E-BP $P^{R N A i}$ treated with rapamycin had fat levels statistically indistinguishable from $y w$ seml. $\mathrm{N}=15 /$ genotype/ treatment a total of three independent experiments (each with $\mathrm{n}=5$ /genotype/treatment). Values of mutants and controls were statistically compared using student's t-test $\left(^{* * *} \mathrm{p}<0.001\right.$, all other comparisons non-significant except $y w$ seml; $N P 1>4 E-B P^{R N A i}$ treated with rapamycin compared to $y w$, which has a p value $<0.001$-comparison not shown in the graph).

https://doi.org/10.1371/journal.pgen.1010082.g001

\section{Reference}

1. Bahuguna S, Atilano M, Glittenberg M, Lee D, Arora S, Wang L, et al. (2022) Bacterial recognition by PGRP-SA and downstream signalling by Toll/DIF sustain commensal gut bacteria in Drosophila. PLoS Genet 18(1): e1009992. https://doi.org/10.1371/journal.pgen.1009992 PMID: 35007276 